\title{
Sternalis muscle in jordanian population: a prevalence study and level of physicians' awareness
}

\author{
Zina M. Al-Alami ${ }^{1}$, Asma’a Al-Mnayyis $^{2}$, Neveen Altamimi $^{3}$ \\ ${ }^{1}$ Department of Medical Laboratory Sciences, Faculty of Allied Medical Sciences, Al-Ahliyya Amman University, Amman, ${ }^{2}$ Department of Clinical \\ Sciences, Faculty of Medicine, Yarmouk University, Irbid, ${ }^{3}$ King Abdullah University Hospital (KAUH), Al-Ramtha, Jordan
}

\begin{abstract}
Sternalis muscle (SM) is an anatomical variant that lies parallel to the sternum. It is present in (8\%) of human population. Awareness about its presence during thoracic imaging is important, since it might be misdiagnosed as a tumor. This study is the first that discusses the prevalence of SM in the Jordanian population and document the level of awareness about SM among intern doctors and surgery and radiology residents. Our aims are to know the prevalence of SM in the Jordanian population, using thoracic multi-detector computerized tomography (CT) images, and to assess the awareness about SM among a sample of intern and resident Jordanian physicians. Random anonymous axial thoracic multi-detector CT images of 1,709 (801 females and 908 males) Jordanian patients, were examined for the presence or absence of unilateral and/ or bilateral SM. A questionnaire aiming to identify SM was distributed among 175 intern doctors, 26 surgery resident and 28 radiology resident doctors, their answers were summarized. The prevalence of SM among Jordanians is 5.9\%. The prevalence of unilateral SM is $2.1 \%$ on the right side of the thorax and $1.9 \%$ on the left side, bilateral prevalence was $1.8 \%$. While $35.7 \%$ of the radiology residents could identify SM using CT and/or anatomy images, only $3.9 \%$ of surgery residents and none of the intern doctors could. We concluded that SM is present in the Jordanian population, with a prevalence of $5.9 \%$ which falls within the global average. Intern doctors and surgery and radiology residents are almost unaware and unfamiliar about SM.
\end{abstract}

Key words: Awareness, Computerized tomography, Jordan, Prevalence, Sternalis

Received February 21, 2020; Revised April 23, 2020; Accepted May 7, 2020

\section{Introduction}

The sternalis muscle (SM) (plural sternales) is considered an anatomical variation. SM is a slim band of muscular fibers that lies parallel to the sternum. It is present in (8\%) of the human population [1]. Its prevalence was studied in different populations; Filipinos [2], Japanese [3], American [4],

\section{Corresponding author:}

Zina Al-Alami (iD

Department of Medical Laboratory Sciences, Faculty of Allied Medical Sciences, Al-Ahliyya Amman University, Amman 19328, Jordan

E-mail:z.alalami@ammanu.edu.jo
Indian [5], Chinese [6, 7] and Korean [8]. Moreover, sternalis' prevalence was also studied in Bulgarian [9]; Thais [10-12] and Chilean [13]. SM prevalence was usually studied using different imaging techniques such as mammography [14], conventional computerized tomography (CT) $[13,15]$ multidetector CT $[6,16]$, sonography $[15]$ and magnetic resonance [17]. Anatomists, clinicians, surgeons and imaging practitioners, should be aware of the possibility of the presence of SM. Awareness about the presence of SM should be considered during thoracic surgery, and during thoracic imaging particularly mammography, as it might be misdiagnosed as a tumor [1]. Surgically, SM as 'muscular flaps' may be used in breast augmentation [18]. This study will enrich the existing literature about $\mathrm{SM}$, since there is no single article

\section{Copyright ( 2020 . Anatomy \& Cell Biology}

This is an Open Access article distributed under the terms of the Creative Commons Attribution Non-Commercial License (http://creativecommons.org/licenses/by-nc/4.0/) which permits unrestricted non-commercial use, distribution, and reproduction in any medium, provided the original work is properly cited. 
that discussed and studied the prevalence of this anatomical variation neither in the Jordanian population nor in the Arab populations. The aims of this study are (a) to know the prevalence of SM in Jordanian population, using thoracic multi-detector CT images, and (b) to study the awareness about SM among a sample of intern and resident Jordanian physicians. Our research questions were: (a) Is the anatomic variant (sternalis) present in the Jordanian population? (b) What is its prevalence, and does it fall within the global average? (c) Would the sample of intern doctors and residents be able to identify SM in unlabeled CT images and/or anatomy figures?

\section{Materials and Methods}

\section{Prevalence of sternalis muscle}

A cross sectional study was performed by using random anonymous multi-detector CT images stored in the Picture Archiving and Communication System at King Abdullah University Hospital (KAUH). A number of 1,709 of multidetector CT images taken for patients from the north of Jordan, were included in the study (801 females and 908 males), for patients born between the years 1916 and 2018. Images were reviewed by a consultant radiologist with more than 10 years experience in thoracic imaging and more than 5 years experience in musculoskeletal imaging.

Axial thoracic multi-detector CT cuts were studied for the identification of the SM, with the analysis of the sagittal and coronal reconstructions when required. The muscle was noticed to be thin or thick, band-like or sheet-like in different images. The CT attenuation value of the identified muscle was measured at multiple points with the muscle using the Hounsfield unit and compared to adjacent muscles to ensure that it is a muscular tissue and not a tissue that mimics muscle, such as thoracic wall tumors or vascular structures when analyzing non-enhanced CT images. To ensure privacy of the images, examined images were given serial numbers and the following data were recorded for every image: the sex, date of birth, and the presence or absence of right SM and/or left SM.

Our inclusion criterium was that the patient should be recorded as Jordanian according to his/her identity card, our exclusion criteria were (a) non- Jordanian patients, (b) severely cachectic patients where the muscle layers can't be discriminated well and (c) the presence of previous breast or upper thoracic surgeries where SM can be affected by the surgery.

\section{Awareness of physicians about sternalis muscle}

A cross sectional study on a group of physicians was performed. A quiz-like questionnaire was prepared (supplementary data), it included one axial CT image showing SM (from one of our cases in the study), and another anatomy figure adopted from a recent publication about the chest anatomy [19]. A 700-bed teaching hospital in the north of Jordan was selected randomly to recruit our physicians' sample from. A total of 229 physicians participated in answering the questionnaire (175 intern doctors, 26 surgery residents and 28 radiology residents). The questionnaires were distributed in person and collected by one of the authors. Our inclusion criteria were (a) the physician should be either an intern doctor, or a surgery or radiology resident, at the time of the study (December 2019), (b) the physicians should be working in the same teaching hospital in the north of Jordan.

Our exclusion criteria were (a) general practitioners, (b) specialist radiologists or surgeons. All distributed questionnaires were marked by one of the authors, with the specific answers: Sternalis or sternalis anterior, being the correct answers. Other vague or nonspecific answers related to muscles or other structures were considered incorrect.

In Jordan, after completing six years medical school, each graduate should complete a year of post-graduate training before starting any residency program. This year is referred to as the internship year, and the trainees are called intern doctors. Residency period ranges from 4-5 years of education and clinical training depending on the specialty. After completing the residency and passing the necessary exit exams, the physician is then recognized as a specialist in a certain medical specialty.

Internship level was selected in this study as the new graduates are expected to have good anatomic knowledge, and the surgery and radiology residents were selected because such specialties are expected to have deeper anatomic knowledge compared to others.

\section{Ethical standards}

The authors assert that all procedures contributing to this work complied with the ethical standards of the relevant national guidelines on human experimentation and with the Helsinki Declaration of 1995, as revised in 2000. An Institutional Review Board (IRB) consent was obtained for experimentation with human subjects from KAUH, the IRB 
number is: $13 / 2 / 3496$.

\section{Results}

\section{Prevalence of sternalis muscle}

In the multi-detector CT images, SM was revealed as a longitudinal structure with parasternal course surrounded by fatty tissue, it was bilateral or unilateral (Fig. 1). The prevalence of SM in the Jordanian population was 5.9\%. The prevalence of bilateral SM was $1.8 \%$ (31 cases out of 1,709 images), $16 / 908$ males (1.8\%), and 15/801 females (1.9\%). The prevalence of unilateral right SM was 2.1\% (36 cases out of 1,709 images), 18/908 males (2.0\%), and 18/801 females (2.3\%). The prevalence of unilateral left SM was 1.9\% (33 cases out of 1,709 images), 21/908 males (2.3\%) and 12/801 females (1.5\%). The results are summarized in Table 1.

\section{Awareness of physicians about sternalis muscle}

In the teaching hospital of choice, the total number of intern doctors was 309, and of the surgery residents was 39 , and of radiology residents was 31. A convenient sample of $229(60.4 \%)$ doctors was recruited to complete the questionnaire (175 intern doctors, 26 surgery residents and 28 radiology residents). Only $35.7 \%$ of the radiology residents and $3.8 \%$ of the surgery residents identified SM correctly, none of the intern doctors could.

The majority of the intern doctors did not provide any answer neither on the CT image nor on the anatomy figure (82.9\% and $94.3 \%$ respectively). While, the majority of the surgery and radiology residents, provided incorrect or non-

Table 1. Prevalence of SM in the jordanian population, given as numbers and percentages

\begin{tabular}{lccc}
\hline & Male $(\mathrm{n}=908)$ & Female $(\mathrm{n}=801)$ & Total $(\mathrm{n}=1,709)$ \\
\hline Right SM & $18(2.0)$ & $18(2.3)$ & $36(2.1)$ \\
Left SM & $21(2.3)$ & $12(1.5)$ & $33(1.9)$ \\
Bilateral SM & $16(1.8)$ & $15(1.9)$ & $31(1.8)$ \\
\hline
\end{tabular}

Values represent the number of cases, values in brackets represent the percentage. SM, sternalis muscle.

Table 2. Summary of the results of SM questionnaire completed by intern doctors and surgery and radiology residents

\begin{tabular}{|c|c|c|c|}
\hline Type & Intern doctor & Surgery resident & Radiology resident \\
\hline Total number in the teaching hospital & 309 & 39 & 31 \\
\hline Doctors who answered the questionnaire & $175(56.6)$ & $26(66.7)$ & $28(90.3)$ \\
\hline \multicolumn{4}{|l|}{ Correct answer } \\
\hline CT scan & $0(0.0)$ & $1(3.8)$ & $10(35.7)$ \\
\hline Anatomy & $0(0.0)$ & $1(3.8)$ & $10(35.7)$ \\
\hline \multicolumn{4}{|l|}{ Incorrect or nonspecific answer } \\
\hline CT scan & $29(16.6)$ & $22(84.6)$ & $12(42.9)$ \\
\hline Anatomy & $9(5.1)$ & $15(57.7)$ & $9(32.1)$ \\
\hline \multicolumn{4}{|l|}{ No answer } \\
\hline CT scan & $145(82.9)$ & $3(11.5)$ & $6(21.4)$ \\
\hline Anatomy & $165(94.3)$ & $10(38.5)$ & $9(32.1)$ \\
\hline
\end{tabular}

Values are presented as number only or number (\%). SM, sternalis muscle; CT, computerized tomography.
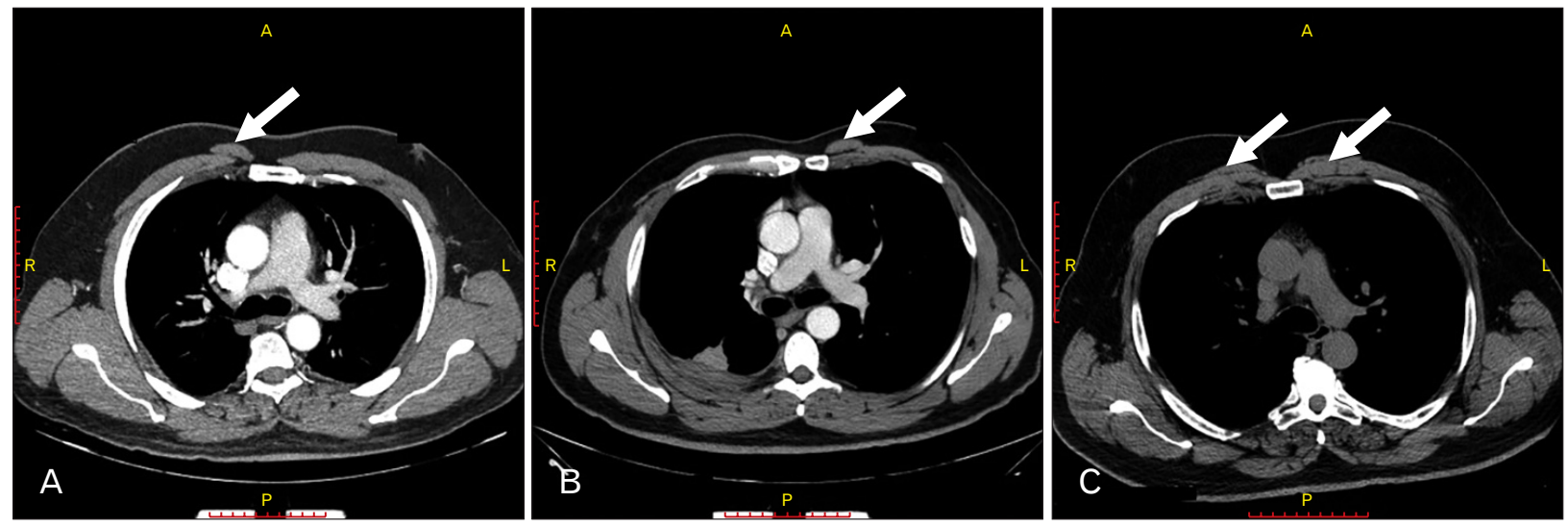

Fig. 1. Multi-detector computerized tomography images showing sternalis muscle (white arrows) on (A) the right of thorax, (B) the left side of thorax and $(\mathrm{C})$ both sides of the thorax. A, anterior; L, left; P, posterior; R, right. 
Table 3. A list of incorrect or nonspecific answers after reading the CT image, the structures are ordered from the highest percentage to the lowest percentage

\begin{tabular}{lc}
\hline \multicolumn{1}{c}{ Structure } & Value $(\mathrm{n}=63)$ \\
\hline Pectoralis major & $19(30.2)$ \\
\hline Pectoralis & $12(19.1)$ \\
\hline Mass or tumor & $11(17.5)$ \\
\hline Rectus abdominis & $4(6.4)$ \\
\hline Lump/breast lump & $3(4.8)$ \\
Muscle & $3(4.8)$ \\
\hline Serratus anterior & $2(3.2)$ \\
\hline Lipoma & $2(3.2)$ \\
\hline Hernia & $2(3.2)$ \\
Normal extra muscle or normal muscle variant & $1(1.6)$ \\
\hline Chest wall muscle & $1(1.6)$ \\
\hline Hematoma & $1(1.6)$ \\
\hline Subcutaneous hematoma & $1(1.6)$ \\
\hline Rectus & $1(1.6)$ \\
\hline Total & $63(100.0)$ \\
\hline
\end{tabular}

Values represent the number of physicians who gave that specific incorrect answer, values in brackets represent the percentage. CT, computerized tomography.

specific answers. The results are summarized in Table 2.

Overall, when physicians' incorrect or nonspecific answers were summarized, different percentages were obtained. As illustrated in Tables 3 and 4, the majority labelled $\mathrm{SM}$ in the CT scan as pectoralis major (30.2\%), and misread the anatomy figure as pectoralis major $(33.3 \%)$ or pectoralis $(33.3 \%)$.

\section{Discussion}

$\mathrm{SM}$ is a tiny muscle found in the anterior pectoral region, it is positioned superficial to the pectoralis major muscles and to the sternum itself [20]. Although the knowledge of the presence, familiarity, awareness and the prevalence of SM as an anatomical variation is of great importance, many physicians and medical students were unfamiliar with SM [21].

Using cadavers and/or imaging modalities, sternalis' prevalence was documented and showed great variation among geographic populations. The lowest prevalence ever recorded was in the Chilean population (0.87\%) [13] and in the Taiwanese population (less than 1.00\%) [22], the highest occurrence percentage (23.5\%) was recorded in northern Chinese population [9]. In their review article Snosek et al. [23] reported and documented texts and tables showing that studies on cadavers on Asian, African and European populations showed that its general prevalence is $7.8 \%$. Studies based on various imaging modalities and surgical procedures
Table 4. A list of incorrect or nonspecific answers after reading the anatomy figure, the structures are ordered from the highest percentage to the lowest percentage

\begin{tabular}{lc}
\hline \multicolumn{1}{c}{ Structure } & Value $(\mathrm{n}=33)$ \\
\hline Pectoralis & $11(33.3)$ \\
\hline Pectoralis major & $11(33.3)$ \\
\hline Rectus & $5(15.2)$ \\
\hline Pectoralis minor & $2(6.1)$ \\
\hline Normal extra muscle or normal muscle variant & $1(3.0)$ \\
Muscle & $1(3.0)$ \\
Scalene muscle & $1(3.0)$ \\
\hline Conjoint tendon & $1(3.0)$ \\
\hline Total & $33(100.0)$ \\
\hline
\end{tabular}

Values represent the number of physicians who gave that specific incorrect answer, values in brackets represent the percentage.

showed a prevalence of $0.6 \%$, yet, mammographs showed a prevalence of $0.02 \%$, while studies using multi-detector CT showed $6.4 \%$ prevalence. As to complete the literature review until 2020, we have summarized the published data related to the prevalence of SM in populations since 2014 (Table 5) [6, 13, 24-27], in our review we have used pubmed and google scholar.

According to the official Jordanian department of statistics (http://dosweb.dos.gov.jo/), the number of the Jordanian population by February 2020 was approximately 10,580,425 persons. Therefore, a sample size of 384 cases would be enough for this study; nevertheless, we have recruited 1,709 cases of multi-detector CT images for this study. Our results were in accordance to previous results, that showed that SM presents with equal frequency in a right sided $(2.1 \%)$ or left sided (1.9\%) asymmetric form, and that unilateral muscles occur approximately as frequently as bilateral muscles $(1.8 \%)$ [28].

Our study represents a new record, reporting the prevalence of SM in the Jordanian population. This will enrich the existing literature about SM, since there is no single article that discussed and studied the prevalence of this anatomical variation neither in the Jordanian population nor in the Arab populations.

We are aware that recognizing this anatomic variant is very important for anatomists and clinicians in general and during radiological and surgical residencies in particular, to avoid misdiagnosing this muscular tissue as tumor when reading thoracic or breast images or when performing thoracic surgeries, and to get the benefit of using it as a flap in some surgeries. SM mimics breast cancer mass using mammography, accordingly, it might be misdiagnosed or incorrectly understood as a mass requiring surgical removal [14], 
Table 5. Sternalis prevalence in the period (2014-2020)

\begin{tabular}{llccc}
\hline \multicolumn{1}{c}{ Population } & \multicolumn{1}{c}{ Cadaver or imaging modality } & Year of publication & Prevalence (\%) & Reference \\
\hline Caldas (Colombia) & Cadavers & 2019 & 2.94 & {$[24]$} \\
South African & Cadavers & 2018 & 2.25 & {$[25]$} \\
Chilean & axial computerized tomography & 2017 & 0.87 & {$[13]$} \\
Indian-Eastern delta region of Tamil Nadu & Cadavers & 2016 & 3.3 & {$[26]$} \\
Indian-South Gujarat Region & Cadavers & 2016 & 5 & {$[27]$} \\
Chinese & Multi-detector computed tomography & 2014 & 5.8 & {$[6]$} \\
\hline
\end{tabular}

or it might be misdiagnosed as a hernia in pectoralis muscle [18]. Clinically, SM can also be used to cover the prosthesis in the breast, in augmentation mammoplasties [29], or used as a flap in reconstructive myocutaneous flaps [9]. Surprisingly, our study showed that many of the physicians who provided incorrect and nonspecific answers misdiagnosed SM as a tumor or mass, breast lump, lipoma, hernia or hematoma, particularly when reviewing the provided CT image.

Actually, after completing the first part of our study, and knowing that SM prevalence falls within the global range (5.9\%), we decided to pursue our work to check if the intern doctors (as a newly graduate physicians with fresh anatomic knowledge) and the surgery and radiology residents (whom were selected because such specialties are expected to have deeper anatomy knowledge compared to others) are aware and familiar about the identification of SM using CT images and anatomy figures. Taking in consideration that, SM was never mentioned in any of the hospital radiology reports of the 1,709 CT images that were included in the current study. Unfortunately, our results showed that only about one third of radiology residents and a very low percentage of surgery residents identified SM correctly, yet none of the intern doctors could.

A general review of literature showed that there is only single study that was published in 1999 which reported results of a survey to assess the familiarity about SM, the study revealed that there was a near-universal unfamiliarity of SM among faculty members, medical students and general and plastic surgery residents [21]. Our results demonstrated that anatomic variants are easily misdiagnosed or ignored in medical reports. Therefore, this highlights the fact that it is crucial to introduce and concentrate on anatomic variants to medical students especially in their first years of medical school. Moreover, clinical rotations across different specialties should also focus on different anatomic variants.

Future work might include classifying the types SM according to the existing known classifications, and documenting sternalis variants if present. Besides, exploring whether other anomalies, diseases or variations are likely to accompany the presence of SM. Other future work might include studying the prevalence of SM in more expanded sample of the Jordanian or Arabic populations using various radiological modalities. And also, evaluating the contents related to anatomic variants in anatomy and training syllabi in the medical education years. One weakness point in this research might be the existence of SM CT image and anatomy figure in the same questionnaire, (supplementary data), smart doctors would have noticed that they were asking about the same structure, so this might be a limiting point about the evaluation of their knowledge of the sternalis.

In conclusion, we reported a previously undocumented prevalence of SM in a sample of Jordanian population. SM is present in the Jordanian population, its prevalence (5.9\%) falls within the global average. Intern doctors and surgery and radiology residents are almost unfamiliar about SM.

\section{ORCID}

Zina M. Al-Alami: https://orcid.org/0000-0003-3108-6022 Asma'a Al-Mnayyis:

https://orcid.org/0000-0002-3800-7641

Neveen Altamimi:

https://orcid.org/0000-0003-4483-646X

\section{Author Contributions}

Conceptualization: ZMA. Data acquisition: AA. Data analysis or interpretation: AA, NA. Drafting of the manuscript: ZMA. Critical revision of the manuscript: ZMA, AA, NA. Approval of the final version of the manuscript: all authors.

\section{Conflicts of Interest}

No potential conflict of interest relevant to this article was reported. 


\section{Acknowledgements}

This work was published with the support of Al-Ahliyya Amman University. The authors acknowledge Al-Ahliyya Amman University and Yarmouk University for their support.

\section{References}

1. Pinhal-Enfield G, Varricchio P, DeFouw D, Vasan NS. Sternalis muscle: importance of its awareness in chest imaging and clinical significance. Int J Anat Var 2011;4:106-8.

2. Yap SE. Musculus sternalis in Filipinos. Anat Rec 1921;21:35372.

3. Taniguchi T, Tochihara J. Studies of the sternalis muscle in Japanese (No. 2), with special references to fetus and anencephalon of Japanese. Acta Anat Jpn 1932;7:1232-49.

4. Barlow RN. The sternalis muscle in American whites and Negroes. Anat Rec 1935;61:413-26.

5. Kacker GN. Sternalis muscle in UP Indian subjects. J Anat Soc India 1960;9:101-3.

6. Ge Z, Tong Y, Zhu S, Fang X, Zhuo L, Gong X. Prevalence and variance of the sternalis muscle: a study in the Chinese population using multi-detector CT. Surg Radiol Anat 2014;36:219-24.

7. Shoumin YU. Observations on the musculus sternalis in Chinese. Acta Anat Sin 1954;(2).

8. Kim WS, Kim SI, Han SR. Three cases of sternalis muscle in Korean. Korean J Phys Anthropol 2000;13:337-44.

9. Jelev L, Georgiev G, Surchev L. The sternalis muscle in the Bulgarian population: classification of sternales. J Anat 2001;199(Pt 3):359-63.

10. Chaijaroonkhanarak W, Amarttayakong P, Pannangron W, Umka J, Namking M, Chaisiwamongkol K, Kondo H, Prachaney P. Incidence of the sternalis muscle in Northeastern Thais. Srinagarind Med J 2013;28:62-5.

11. Kirirat P, Thaweethamsewee P, Subhadhirasakul P, Puriwathanakul K, Tanomkiat W. The sternalis muscle in the Thai population. J Health Sci Med Res 2005;23:255-9.

12. Plakornkul V, Viravud Y. Sternalis muscle: anatomical variations in Thais. Siriraj Med J 2012;64(suppl):19-21.

13. Molina CR, Pinochet JA, Heras AA, Taunton MJ, Letelier RG, Letelier RF. Prevalence of the sternalis muscle in Chilean population: a computed tomography study. Ital J Anat Embryol 2017;122:173-8.
14. Bradley FM, Hoover HC Jr, Hulka CA, Whitman GJ, McCarthy KA, Hall DA, Moore R, Kopans DB. The sternalis muscle: an unusual normal finding seen on mammography. AJR Am J Roentgenol 1996;166:33-6.

15. Nuthakki S, Gross M, Fessell D. Sonography and helical computed tomography of the sternalis muscle. J Ultrasound Med 2007;26:247-50.

16. Kobayashi S, Tomizawa H, Tomizawa Y, Manaka Y. Incidental finding of the sternalis muscle on chest multidetector-row computed tomography (MDCT): the diagnostic value of additional postprocessed MDCT images for an uncommon muscular variant. Intern Med 2013;52:1137-9.

17. Gruber L, Martinoli C, Tagliafico AS, Gruber J, Klauser AS. A rare case of a symptomatic sternalis muscle: ultrasonograpy and MRI correlation. Ultrasound Int Open 2016;2:E140-1.

18. Georgiev GP, Jelev L, Ovtscharoff VA. On the clinical significance of the sternalis muscle. Folia Med (Plovdiv) 2009;51:53-6.

19. Saxena AK, Alalayet YF. Surgical anatomy of the chest wall. In: Saxena AK, editor. Chest wall deformities. Berlin: Springer; 2017. p. 37-53.

20. Arráez-Aybar LA, Sobrado-Perez J, Merida-Velasco JR. Left musculus sternalis. Clin Anat 2003;16:350-4.

21. Bailey PM, Tzarnas CD. The sternalis muscle: a normal finding encountered during breast surgery. Plast Reconstr Surg 1999;103:1189-90.

22. Jeng $\mathrm{H}, \mathrm{Su}$ SJ. The sternalis muscle: an uncommon anatomical variant among Taiwanese. J Anat 1998;193(Pt 2):287-8.

23. Snosek M, Tubbs RS, Loukas M. Sternalis muscle, what every anatomist and clinician should know. Clin Anat 2014;27:86684.

24. Duque-Parra JE, Barco-Ríos J, Vélez-García JF. Incidence of sternalis muscle in the Caldas population (Colombia): anatomical variations. Int J Morphol 2019;37:1342-6.

25. Davimes JG, Bacci N, Mazengenya P. Evidence of the sternalis muscle in two South African cadavers. Surg Radiol Anat 2018;40:1313-7.

26. Puthuraj MP, Shanmugam S. Prevalence of rectus sternalis: a clinical enigma. Indian J Basic Appl Med Res 2016;5:5-10.

27. Parmar ND, Gupta DS. A study of morphological variation of pectoral region muscle 'Rectus Sternalis' in south Gujarat region. Int J Anat Res 2016;4:2423-28.

28. Hung L, Lucaciu OC, Wong JJ. Back to the debate: sternalis muscle. Int J Morphol 2012;30:330-6.

29. Khan UD. Use of the rectus sternalis in augmentation mammoplasty: case report and literature search. Aesthetic Plast Surg 2008;32:21-4. 